\title{
Analysis of Clinical Characteristics, Radiological Predictors, Pathological Features, and Perioperative Outcomes Associated with Perinephric Fat Adhesion Degree
}

\author{
Junqiang Liu $\mathbb{D}^{1},{ }^{1}$ Yiheng Jiang $\mathbb{D}^{1},{ }^{1}$ Hongwei Huang $\mathbb{D}^{1},{ }^{1}$ Zheng Zhu $\mathbb{D}^{2},{ }^{2}$ Jing Chen $\left(\mathbb{D},{ }^{3}\right.$ \\ Dikuan Liu $\mathbb{D}^{1},{ }^{1}$ Lina Wang $\mathbb{D}^{1},{ }^{1}$ Huafeng Zong $\mathbb{D}^{4},{ }^{4}$ Xishuang Song $\mathbb{D}^{1},{ }^{1}$ Xuejian Wang $\mathbb{D}^{1}$, \\ Xinqing Zhu $\mathbb{D}$, ${ }^{1}$ Jianbo Wang $\mathbb{D}^{1},{ }^{1}$ Sony Nahayo $\left(\mathbb{D},{ }^{1}\right.$ Qiwei Chen $\mathbb{D}^{1},{ }^{1,5}$ and Deyong Yang $\mathbb{D}^{1}$ \\ ${ }^{1}$ Department of Urology, First Affiliated Hospital of Dalian Medical University, Dalian 116021, China \\ ${ }^{2}$ Department of Internal Medicine, University of California, Davis, USA \\ ${ }^{3}$ Department of Imaging, First Affiliated Hospital of Dalian Medical University, Dalian 116021, China \\ ${ }^{4}$ Department of Pathology, Dalian Friendship Hospital, Dalian 116021, China \\ ${ }^{5}$ School of Information Science and Technology of Dalian Maritime University, 116000 Dalian City, Liaoning Province, China
}

Correspondence should be addressed to Qiwei Chen; chenqiwei@dmu.edu.cn and Deyong Yang; yangdeyong@dmu.edu.cn

Received 28 June 2021; Accepted 1 December 2021; Published 27 December 2021

Academic Editor: Reza Izadpanah

Copyright (C) 2021 Junqiang Liu et al. This is an open access article distributed under the Creative Commons Attribution License, which permits unrestricted use, distribution, and reproduction in any medium, provided the original work is properly cited.

Background. To assess the clinical characteristics, radiological predictors, and pathological features of perinephric fat adhesion degree (PFAD) graded based on fixed criteria and to determine the impact of adherent perinephric fat (APF) on retroperitoneal laparoscopic partial nephrectomy (RLPN) outcomes. Methods. 84 patients undergoing RLPN were included and graded into 4 groups based on PFAD. Univariate and multivariate analyses were performed for clinical characteristics and radiological predictors of PFAD. Perioperative data were compared between APF groups and non-APF groups. Masson staining determined collagen fibers. Immunohistochemistry detected CD45 immune cells and CD34 vessels. Results. 20, 28, 18, and 18 patients were graded as normal perinephric fat (NPF), mild adherent perinephric fat (MiPF), moderate adherent perinephric fat (MoPF), and severe adherent perinephric fat (SPF), respectively. Multivariate analysis revealed that gender $(p<0.001)$, age $(p=0.003)$, and hypertension $(p=0.006)$ were significant clinical risk factors of PFAD, while radiological predictors included perinephric stranding $(p=0.001)$, posterior perinephric fat thickness $(p=0.009)$, and perinephric fat density $(p=0.02)$. APF was associated with drain output $(p=0.012)$ and accompanied by immune cells gathering in renal cortex near thickened renal capsule with many vessels. Conclusions. Clinical characteristics and radiological predictors can evaluate PFAD and may assist to guide preoperative surgical option. Pathological features of APF reflect decapsulation and bleeding during kidney mobilization at RLPN.

\section{Introduction}

For T1 stage renal cell carcinoma, especially for T1a tumors, partial nephrectomy is recognized as the standard operation whenever technically feasible [1-3]. In RLPN, multiple radiological scores that may anticipate tumor complexity are proposed to describe the tumor-specific characteristics, but they neglect other factors. One of the potential patientspecific factors for surgical complexity is APF characterized by thickening and adhesion fat surrounding the kidneys [4].

Due to lack of consensus objective grading criteria for the definition of APF, the incidence of APF varies greatly, ranging from $10.6 \%$ to $55.2 \%$ according to the previous studies [4, 5]. Most researchers conducted retrospective analysis based solely on the description of APF adhesion in surgical records [6-8]. Zheng et al. [9] graded APF according to the operative time for renal mobilization. Dariane et al. 
[10] graded APF based on the subjective surgical score for decapsulation during dissection. Despite these attempts to improve objectivity, there appears to be no clear criteria to grade PFAD.

So far, the pathogenesis of APF remains unclear. At present, the mainstream argues that APF has a positive correlation with chronic inflammation. Previous studies showed interleukins and adipocyte size were increased in APF $[10,11]$. But these findings failed to reflect the clinical, radiological, and surgical characteristics of APF.

Therefore, our study established objective criteria after multiple surgical videos were reviewed. The present study is to prospectively assess patients' clinical characteristics and radiological predictors for PFAD graded according to objective criteria and to analyze the impact of APF on RLPN outcomes and pathological features of APF. Moreover, we explored the relationship between clinical characteristics, imaging findings, and pathology of APF.

\section{Methods}

2.1. Patients and Data Collection. We performed a prospective study of patients who underwent RLPN for suspicious renal mass between March 2017 and March 2018 at the First Affiliated Hospital of Dalian Medical University. The study was conducted according to the guidelines of the Declaration of Helsinki after approval from our hospital's institutional ethical committee (First Affiliated Hospital of Dalian Medical University). The clinical variables (age, gender, body mass index (BMI), preoperative eGFR, hypertension, diabetes, active smoking, and alcoholism), perioperative outcomes (operative time (OT), warm ischemia time (WIT), estimated blood loss (EBL), transfusions, Clavien-Dindo classification (CDC), length of stay, drain output, postoperative gastrointestinal recovery time, postoperative fever, gastrointestinal discomfort, and change dressing frequency), and postoperative pathology results (Fuhrman grade) were collected.

2.2. Criteria for PFAD Grading. PFAD is divided into four grades (Figure 1). Normal perinephric fat (NPF): easily blunt dissect perinephric fat from kidney, less fibers connect renal capsule and fat; mild adherent perinephric fat (MiPF): easily blunt dissect, more fibers connect renal capsule and fat, and scattered flaky fat remains on the renal capsule after blunt dissection; moderate adherent perinephric fat (MoPF): part of perinephric fat adheres to the kidney and need sharp dissection; and severe adherent perinephric fat (SPF): all parts of perinephric fat adhere to the kidney and need sharp dissection or subcapsular dissection. NPF and MiPF were defined as non-APF, which did not complicate kidney mobilization while the latter two grades were APF.

2.3. Radiological Data. All patients underwent preoperative abdominal computed tomographic (CT) imaging. The thickness of the medial, lateral, posterior, and poster lateral perinephric fat were measured at the level of the renal vena cava according to the method described by Eisner et al. [12].
Perinephric fat density was a manually selected area close to the renal capsule, and Hounsfield units (HU) in this area were calculated automatically. Perinephric fat area was defined as the area between the medial and posterior renal fat thickness measurement line. Perinephric stranding was determined as Kim et al. [13] described (Figure 2).

The preoperative CT was assessed by two urologists (J.L. and H.H.) blinded to patient PFAD status independently. If there were any differences between the two observers, the corresponding image was assigned to another highly qualified urologist (D.Y.) for final results.

2.4. Histological Analysis. The isolated perinephric fat and adjacent kidney were taken from the NPF and the SPF, fixed in $4 \%$ formalin buffer, embedded in paraffin, and cut into $4 \mathrm{~mm}$ thick serial sections. The slices were stained by the Masson method. The primary antibodies against CD45 $(1: 200)$ and CD34 (1:200) were from Proteintech (China). After staining, the samples were observed under the microscope. Light yellow, brown yellow, or dark brown was supposed as the positive expression. Positive control for Masson staining was defined as renal capsule stained. Positive control for CD34 was defined as blood vessels stained (brown in the cytoplasm). Positive control for CD45 was defined as renal cortex or renal capsule stained.

Besides, an additional file shows the immunodetection methods (Additional file 1). We performed immunohistological staining of CD34, a marker of endothelium (positive in glomerular while negative in kidney tubules), and CD45, a marker of immune cells (positive in lymph nodes while negative in normal kidney tissues) in this research.

2.5. Statistical Analysis. Continuous variables were reported as median (minimum and maximum) and qualitative variables as ratio (percentage). Univariate and ordered multivariate logistic regression analyses were performed to assess clinical characteristics and radiological predictors of PFAD. The chi-square test was used to compare categorical data, and the Mann-Whitney $U$ test was used to compare nonnormally distributed continuous variables and ordered qualitative variables for perioperative outcomes or pathological grading of renal cell carcinoma (RCC) between the patients with APF and non-APF. $P$ values $<0.05$ were considered statistically significant. All statistical analyses were performed using SPSS version 20.0.

\section{Results}

3.1. Patients' Clinical and Radiographic Characteristics. The clinical and radiographic characteristics in our study are given in Table 1 . The PFAD was graded as none, mild, moderate, and severe in $23.8 \%, 33.3 \%, 21.4 \%$, and $21.4 \%$ of our patient cohort, respectively. Of these, $57 \%$ of the patients were over 55 years. The majority of patients were male (61.9\%) and had malignant tumors $(61.9 \%)$. The proportion of patients with malignant tumors and hypertension increases with the PFAD, as well as the median perinephric fat area and perinephric fat thickness. Only 2 patients had a 


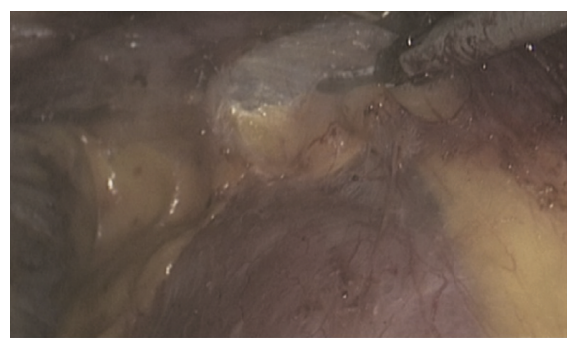

(a)

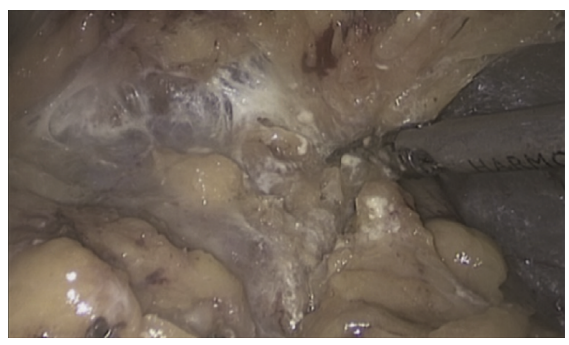

(c)

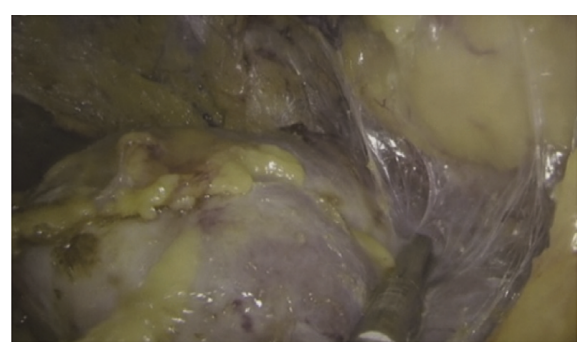

(b)

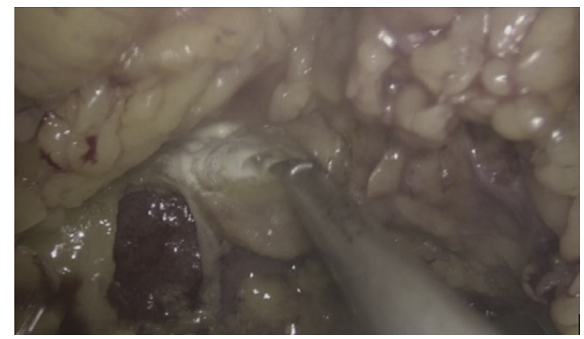

(d)

Figure 1: Surgical criterion of APF. (a) Normal perinephric fat (NPF). (b) Mild adherent perinephric fat (MiPF). (c) Moderate adherent perinephric fat (MoPF). (d) Severe adherent perinephric fat (SPF).

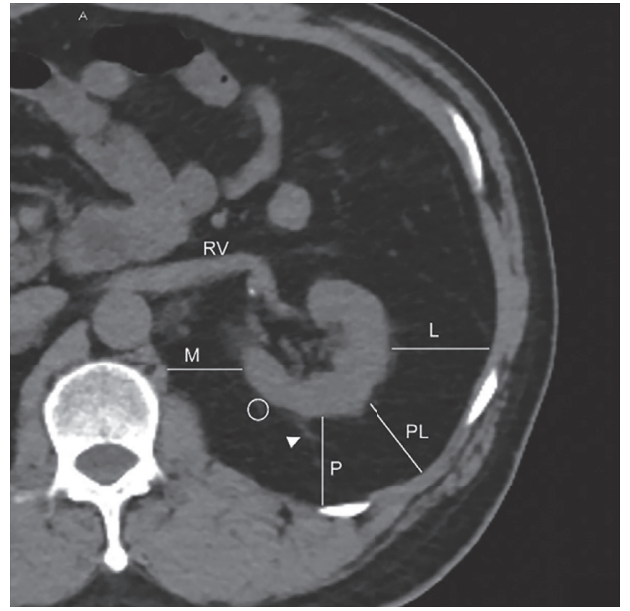

FIGURE 2: Measurement of perinephric fat at the level of the vein. M, medial perinephric fat thickness; L, lateral perinephric fat thickness; P, posterior perinephric fat thickness; PL, posterolateral perinephric fat thickness; circle, $\mathrm{HU}$ of perinephric fat; triangle, stranding; RV, renal vein.

history of chronic nephritis and both were in the SPF group. Proportion of patients with none, mild-to-moderate, and severe perinephric stranding were $61.9 \%, 28.6 \%$, and $9.5 \%$, respectively.

3.2. Clinical Characteristics for PFAD. Table 2 provides the clinical characteristics of PFAD, and bold values indicate significantly correlated factors. PFAD was significantly associated with clinical parameters including increasing age (OR 3.76, $p=0.003$ ), male gender (OR 13.14, $p<0.001$ ), and hypertension (OR 3.28, $p=0.006$ ) on multivariable analysis, whereas other clinical variables were not associated with PFAD.
3.3. Radiological Predictors for PFAD. As given in Table 3, all of radiological variables were found to be predictive of PFAD (all $p<0.001$, marked as bold values) on univariable analysis. However, multivariate analysis showed that perinephric stranding (type 1 OR 25.05, $p=0.001$; type 2 OR 35.21, $p=0.033$ ), posterior fat thickness (OR 11.46, $p=0.009$ ), and perinephric fat density (OR 1.08, $p=0.02$ ) appeared to be the most predictive of PFAD.

3.4. Impact of APF on Perioperative Outcomes and Pathological Grading. The 84 cases comprised 36 with APF (MoPF or SPF) and 48 with non-APF (NPF or MiPF). Drain output is significantly higher in the APF group (169 vs. $125.5 \mathrm{ml}, p=0.012$, marked as bold values; Table 4). Other intraoperative variables (OT, WIT, EBL, and transfusions), postoperative variables (postoperative gastrointestinal recovery time, length of stay, postoperative fever, postoperative gastrointestinal discomfort, change dressing frequency, and CDC), and Fuhrman grading were similar between the two groups $(p>0.05)$.

3.5. Histological Analysis. Masson staining showed that renal capsule was thin and had two layers in the non-APF group. Our study defined the outer membrane-like structure as extracapsular fascia. Patients with APF lack extracapsular fascia and the thickness of the renal capsule increased significantly (Figure 3(a)). Immunohistochemistry showed that CD45+ immune cells accumulate in the renal cortex and infiltrate into renal capsule in the APF group (Figure 3(b)). In patients with APF, the CD34+ vascular endothelial cells were significantly increased and arranged into a vascular-like structure (Figure 3(c)). 
TABLE 1: Clinical and radiographic characteristics stratified by adhesiveness of perinephric fat.

\begin{tabular}{|c|c|c|c|c|c|}
\hline Characteristics & $\operatorname{NPF}(n=20)$ & $\operatorname{MiPF}(n=28)$ & $\operatorname{MoPF}(n=18)$ & SPF $(n=18)$ & Total $(n=84)$ \\
\hline \multicolumn{6}{|l|}{ Age (y) } \\
\hline$\leq 55$ & $12(60)$ & $13(46)$ & $7(39)$ & $4(22)$ & $36(43)$ \\
\hline$>55$ & $8(40)$ & $15(54)$ & $11(61)$ & $14(78)$ & $48(57)$ \\
\hline Male gender (\%) & $6(30)$ & $14(50)$ & $15(83.3)$ & $17(94.4)$ & $52(61.9)$ \\
\hline \multicolumn{6}{|l|}{ BMI, $\mathrm{kg} / \mathrm{m}^{2}(\%)$} \\
\hline$\leq 25$ & $8(40)$ & $15(53.6)$ & $6(33.3)$ & $4(22.2)$ & $33(39.3)$ \\
\hline $25-30$ & $10(50)$ & $9(32.1)$ & $10(55.6)$ & $9(50)$ & $38(45.2)$ \\
\hline$\geq 30$ & $2(10)$ & $4(14.3)$ & $2(11.1)$ & $5(27.8)$ & $13(15.5)$ \\
\hline Diabetes (\%) & $5(25)$ & $6(21.4)$ & $4(22.2)$ & $6(33.3)$ & $19(22.6)$ \\
\hline Hypertension (\%) & $5(25)$ & $12(42.9)$ & $9(50)$ & $12(66.7)$ & $38(45.2)$ \\
\hline Cardiovascular disease (\%) & $3(15)$ & $2(7.1)$ & $3(16.7)$ & $2(11.1)$ & $10(11.9)$ \\
\hline $\begin{array}{l}\text { Preoperative eGFR }(<60 \mathrm{ml} / \\
\min )(\%)\end{array}$ & $0(0)$ & $0(0)$ & $0(0)$ & $2(11.1)$ & $2(2.4)$ \\
\hline Active smoking (\%) & $7(35)$ & $9(32.1)$ & $7(38.9)$ & $8(44.4)$ & $31(36.9)$ \\
\hline Alcoholism (\%) & $7(35)$ & $11(39.3)$ & $6(33.3)$ & $8(44.4)$ & $32(38.1)$ \\
\hline Malignancy (\%) & $10(50)$ & $16(57.1)$ & $12(66.7)$ & $14(77.8)$ & $52(61.9)$ \\
\hline $\begin{array}{l}\text { Preoperative creatinine } \\
(>\mathrm{ULN})(\%)\end{array}$ & $4(20)$ & $3(10.7)$ & $3(16.7)$ & $4(22.2)$ & $14(16.7)$ \\
\hline $\mathrm{HU}$ of perinephric fat & $-103(-106.6,-99.3)$ & $-101(-107.8,-96.3)$ & $-95(-106.6,-94.2)$ & $-88(-98.1,-81.5)$ & $-98(-106.5,-93)$ \\
\hline Perinephric fat area $\left(\mathrm{cm}^{2}\right)$ & $0.69(0.5,1.3)$ & $2.81(1.8,3.3)$ & $5.44(4.5,7.1)$ & $8.61(6.2,12.0)$ & $3.05(1.6,6.2)$ \\
\hline \multicolumn{6}{|l|}{$\begin{array}{l}\text { Perinephric fat thickness } \\
(\mathrm{mm})\end{array}$} \\
\hline Medial & $1(0,3.5)$ & $5.9(3.8,7.7)$ & $7.5(4.9,10.6)$ & $9.6(6.8,17.2)$ & $5.8(3.1,9.2)$ \\
\hline Lateral & $1.1(0,6.3)$ & $10.1(4.9,15.0)$ & $17.5(12.2,23.8)$ & $22.5(8.9,25.3)$ & $11.2(4.3,20.1)$ \\
\hline Posterior & $3.5(2.3,5.3)$ & $7.8(6.0,11.8)$ & $12.7(8.7,16.1)$ & $19.4(16.2,31.4)$ & $9.7(5.7,15.8)$ \\
\hline Posterolateral & $8.8(5.7,11.8)$ & $13.8(9.0,16.9)$ & $15.7(14.0,20.8)$ & $24(19.0,29.6)$ & $15.1(10.2,22.3)$ \\
\hline \multicolumn{6}{|l|}{ Stranding $(\%)$} \\
\hline None & $20(100)$ & $26(92.8)$ & $6(33.3)$ & $0(0)$ & $52(61.9)$ \\
\hline Type 1 & $0(0)$ & $2(7.2)$ & $11(61.1)$ & $11(61.1)$ & $24(28.6)$ \\
\hline Type 2 & $0(0)$ & $0(0)$ & $1(5.6)$ & $7(39.9)$ & $52(9.5)$ \\
\hline
\end{tabular}

TABLE 2: Univariate and multivariable analyses clinical characteristics of PFAD.

\begin{tabular}{lccc}
\hline Characteristics & Univariate & & Multivariate \\
& OR & $P$ value & OR \\
Age & $2.61(1.17,5.82)$ & $\mathbf{0 . 0 1 9}$ & $3.76(1.57,8.97)$ \\
Male gender & $8.39(3.32,21.24)$ & $<\mathbf{0 . 0 0 1}$ & $13.14(4.87,35.46)$ \\
BMI & & & \\
$\quad 2.65(0.82,8.54)$ & 0.103 & \\
$\quad 25-30$ & $1.58(0.68,3.68)$ & 0.375 & \\
Diabetes & $1.83(0.73,4.62)$ & 0.200 & $3.28(1.40,7.65)$ \\
Hypertension & $2.85(1.28,6.33)$ & 0.010 & \\
Cardiovascular disease & $0.99(0.30,3.25)$ & 0.475 & $\mathbf{0 . 0 0 6}$ \\
Active smoking & $1.34(0.60,298)$ & 0.685 & \\
Alcoholism & $1.18(0.53,2.60)$ & 0.065 & \\
Malignancy & $2.14(0.95,4.80)$ & 0.747 & \\
Preoperative creatinine & $0.84(0.30,2.37)$ & & \\
\hline
\end{tabular}

Bold values indicate significantly correlated factors.

\section{Discussion}

APF is one of potential tumor-specific factors that can complicate partial nephrectomy $(\mathrm{PN})$ and is associated with decreased progression-free survival in patients with localized renal cancer $[6,10,14-16]$. Previous studies on the association of APF with clinical, radiological features, or perioperative outcomes are given in Table 5. Due to lack of objective criteria for APF, the incidence of APF varies greatly. Therefore, our study established objective criteria after multiple surgical videos were reviewed. In this study, the incidence of APF was $42.8 \%$, which was between the documented incidence $[6,8,10]$.

Most literatures have confirmed that APF is associated with advanced age and common in males [4-6, 8-10, 14], which corresponds with our study. Male patients have more perinephric fat, whereas women have more subcutaneous fat $[12,17]$. However, in the age, adipose tissue redistributes 
TABLE 3: Univariate and multivariable analyses of predicting factors of PFAD.

\begin{tabular}{lccr}
\hline Characteristics & Univariate & & Multivariate \\
\hline Perinephric fat thickness & OR & $P$ value & OR value \\
$\quad$ Medial & $12.48(4.79,32.55)$ & $<\mathbf{0 . 0 0 1}$ & \\
Lateral & $3.18(2.01,5.04)$ & $<\mathbf{0 . 0 0 1}$ & \\
Posterior & $31.09(10.62,91.02)$ & $<\mathbf{0 . 0 0 1}$ & $11.46(1.86,70.60)$ \\
Posterolateral & $6.68(3.43,13.01)$ & $<\mathbf{0 . 0 0 1}$ & $\mathbf{0 . 0 0 9}$ \\
Perinephric fat area & $1.91(1.55,2.35)$ & $<\mathbf{0 . 0 0 1}$ & \\
HU of perinephric fat & $1.14(1.08,1.20)$ & $<\mathbf{0 . 0 0 1}$ & \\
Stranding & & $<.01,1.14)$ \\
Type 1 & $113.44(21.08,610.32)$ & $<\mathbf{0 . 0 0 1}$ & $25.05(3.76,167.00)$ \\
Type 2 & $986.26(68.87,14123.33)$ & $<\mathbf{0 . 0 0 1}$ & $35.21(1.32,937.66)$ \\
\hline
\end{tabular}

Bold values indicate significantly correlated factors.

TABle 4: Correlation between APF and perioperative outcomes and pathological grading of RCC.

\begin{tabular}{|c|c|c|c|}
\hline Variable & APF & Control group with no APF & $P$ value \\
\hline Operative time (OT) (min) & $110.50(70.8,126.0)$ & $89(85.3,141.5)$ & 0.141 \\
\hline Warm ischemia time (WIT) ( $\mathrm{min})$ & $20.50(17.8,32.0)$ & $21(18.8,25.3)$ & 0.986 \\
\hline Drain output $(\mathrm{ml})$ & $169(121.8,186.3)$ & $125.5(94.5,155.0)$ & 0.012 \\
\hline Postoperative gastrointestinal recovery time (days) & $5(2.8,6.0)$ & $4(3.0,5.0)$ & 0.611 \\
\hline Length of stay (days) & $15(13.8,18.3)$ & $14(12.8,15.3)$ & 0.091 \\
\hline Transfusions & $2(5.6 \%)$ & $3(6.3 \%)$ & 0.894 \\
\hline Estimated blood loss (EBL) (ml) & $240(195.5,282.0)$ & $215(171.5,270.8)$ & 0.249 \\
\hline Postoperative fever $\left(>38.0^{\circ} \mathrm{C}\right)$ & $1(2.8 \%)$ & $4(8.3 \%)$ & 0.287 \\
\hline Postoperative gastrointestine discomfort & $11(30.6 \%)$ & $10(20.8 \%)$ & 0.309 \\
\hline Change dressing frequency & $4.5(3.0,6.3)$ & $3(2.0,5.0)$ & 0.052 \\
\hline Clavien-Dindo classification (CDC) & $18(50 \%)$ & $18(37.5 \%)$ & 0.265 \\
\hline Clavien 1 & $15(41.7 \%)$ & $15(31.3 \%)$ & \\
\hline Clavien 2 & $3(8.3 \%)$ & $3(6.3 \%)$ & \\
\hline Fuhrman grade & $26(72 \%)$ & $26(54 \%)$ & 0.106 \\
\hline I & $10(27.8 \%)$ & $10(20.8 \%)$ & \\
\hline II & $10(27.8 \%)$ & $11(22.9 \%)$ & \\
\hline III & $2(5.6 \%)$ & $4(8.3 \%)$ & \\
\hline IV & $4(11.1 \%)$ & $1(2.1 \%)$ & \\
\hline
\end{tabular}

Bold values indicate significant difference.

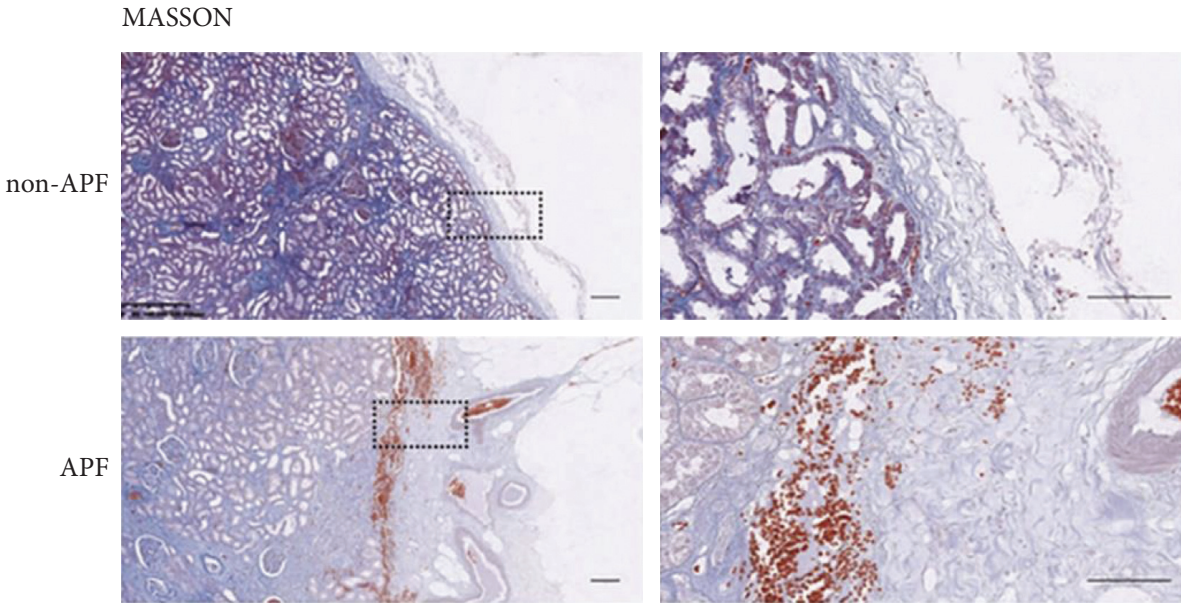

(a)

FIgURE 3: Continued. 


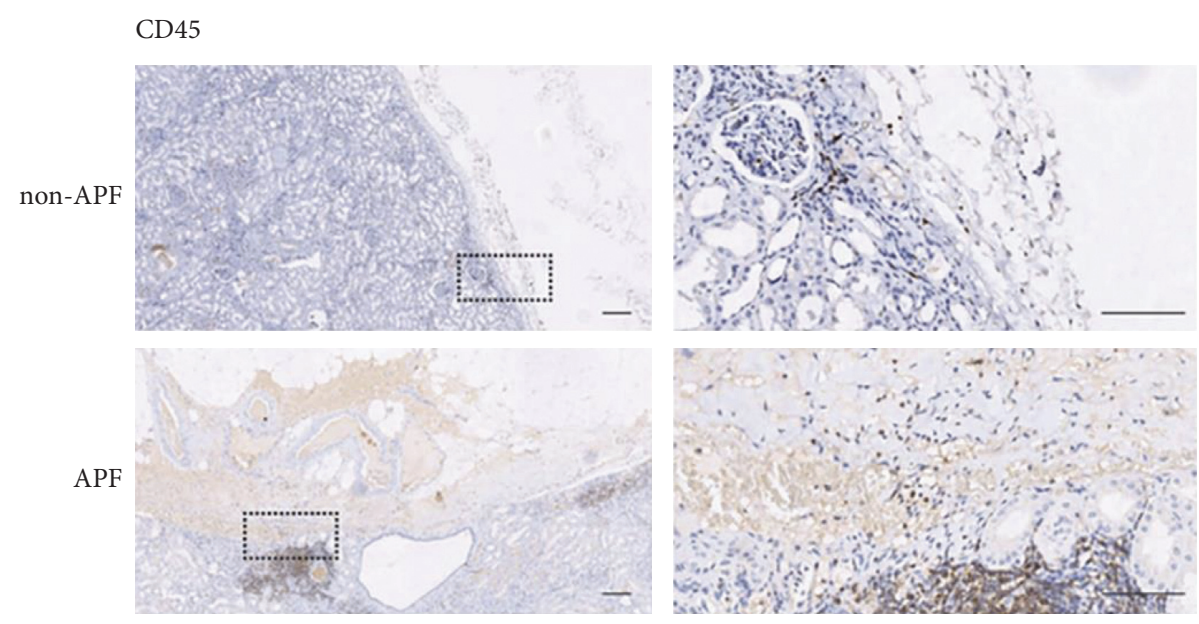

(b)
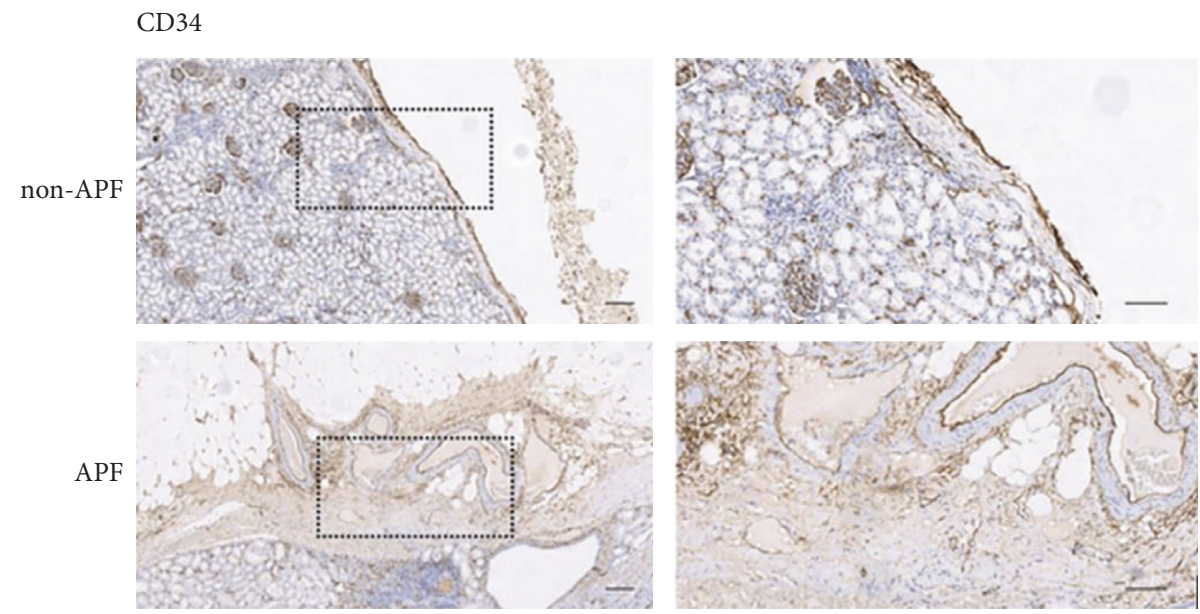

(c)

FiguRE 3: Representative examples of Masson, CD45, and CD34 staining. (a) Non-APF group having a thin extracapsular fascia, APF group renal capsule, and extracapsular fascia fusion thickening. (b) APF CD45 immune cells in the group highly expressed in the renal capsule and adjacent renal cortex. (c) APF group CD34 for vascular staining. Scale bar $=100 \mathrm{~mm}$.

from subcutaneous to visceral and ectopic fat, especially along the kidneys, liver, and bone marrow. Adipose tissue is an endocrine organ which produces hormones such as cytokines especially in tumor necrosis factor- $\alpha$ (TNF $\alpha)$ and interleukin (IL-6). They can increase with aging and excessive accumulation of fat [18]. It was confirmed that the APF group solely increased the expression of sIL-6R, suggesting that APF may be a pathological procedure caused by systemic chronic inflammation [11, 19-21]. Therefore, both age and gender can affect the distribution of visceral and subcutaneous fat and also be the risk factors of APF.

In addition, our study also confirmed hypertension is the risk factor of PFAD, as previous studies reported [22, 23]. The immune system plays an important role in the development of hypertension, and renal immune cell infiltration has been demonstrated in both experimental and clinical hypertension [24]. In the SPF group, we first found that a large number of CD45+ immune cells accumulate in the renal cortex near renal capsule. There are also scattered immune cells, increased blood vessel distribution, and thickening of the renal capsule near renal cortex. Hypertension may affect the adhesion of the renal capsule to the perinephric fat by inducing renal immune cell infiltration. Two patients with previous nephritis were in the SPF group, adding weight to the theory that chronic inflammation of the kidney can affect fat adhesion $[11,25,26]$.

Perinephric stranding and thickness of posterior perinephric fat are important radiological predictors for APF in this study. Perinephric stranding represents a chronic inflammatory reaction which is considered as an important factor for formation of APF. The posterior perinephric fat thickness represents excessive and dysfunctional adipose tissue. Based on the two variables, Mayo adhesive probability (MAP) score was established to predict APF [5], which has been validated in different surgical methods for renal cancer $[6,8,10,14,23]$.

However, the predictive value for perinephric fat density is still controversial. Bylund et al. [4] found that the renal hilum level fat density had no significant effect on APF. Zheng et al. [9] pointed out that perinephric fat surface 
TABLE 5: Summary of previous studies on APF.

\begin{tabular}{|c|c|c|c|c|c|c|}
\hline $\begin{array}{l}\text { Author } \\
\text { (year) }\end{array}$ & Patient & Surgery & APF grading & $\begin{array}{l}\mathrm{APF} \\
\text { rate } \\
(\%)\end{array}$ & Included factors & Significant variables \\
\hline $\begin{array}{l}\text { Bylund et al. } \\
\text { (2013) }\end{array}$ & 29 & $\begin{array}{l}\text { RPN, OPN, or } \\
\text { laparoscopic } \\
\text { cryoablation }\end{array}$ & Operative records & 55.2 & $\begin{array}{l}\text { Clinical, imaging, } \\
\text { pathological, } \\
\text { outcome }\end{array}$ & $\begin{array}{l}\text { Male gender, tumor size, stranding, } \\
\text { tumor }>50 \% \text { exophytic, thickness of } \\
\text { perinephric fat, and OT }\end{array}$ \\
\hline $\begin{array}{l}\text { Zheng et al. } \\
(2014)\end{array}$ & 41 & OPN & $\begin{array}{l}\text { Time of } \\
\text { perinephric fat } \\
\text { dissection on }\end{array}$ & 53.7 & $\begin{array}{l}\text { Clinical, imaging, } \\
\text { pathological, } \\
\text { outcome }\end{array}$ & Male gender and PnFSD \\
\hline $\begin{array}{l}\text { Davidiuk } \\
\text { et al. }(2014)\end{array}$ & 100 & $\mathrm{RPN}$ & $\begin{array}{l}\text { Described by Kim } \\
\text { et al. }\end{array}$ & 30 & $\begin{array}{l}\text { Clinical, imaging, } \\
\text { pathological, } \\
\text { outcome }\end{array}$ & $\begin{array}{c}\text { Male gender, BMI, posterolateral and } \\
\text { posterior perinephric fat, and } \\
\text { stranding }\end{array}$ \\
\hline $\begin{array}{l}\text { Davidiuk } \\
\text { et al. (2015) }\end{array}$ & 100 & RPN & $\begin{array}{l}\text { Described by Kim } \\
\text { et al. }\end{array}$ & 30 & Outcome & \\
\hline $\begin{array}{l}\text { Kobayashi } \\
\text { et al. }(2016)\end{array}$ & 47 & LPN or RALPN & Operative records & 14.9 & $\begin{array}{l}\text { Clinical, imaging, } \\
\text { outcome }\end{array}$ & OT, hypertension, and FSPA on CT \\
\hline $\begin{array}{l}\text { Martin et al. } \\
\text { (2016) }\end{array}$ & 86 & OPN & Operative records & 50.0 & $\begin{array}{c}\text { Clinical, imaging, } \\
\text { outcome }\end{array}$ & Age and MAP score \\
\hline $\begin{array}{l}\text { Kocher et al. } \\
(2016)\end{array}$ & 245 & LPN or RPN & Operative records & 10.6 & $\begin{array}{l}\text { Clinical, imaging, } \\
\text { pathological, } \\
\text { outcome }\end{array}$ & $\begin{array}{c}\text { Age, male gender, stranding, posterior } \\
\text { fat thickness, MAP score, malignant } \\
\text { renal histology, operating time, and } \\
\text { EBL }\end{array}$ \\
\hline $\begin{array}{l}\text { Dariane et al. } \\
\text { (2016) }\end{array}$ & 125 & RPN or OPN & $\begin{array}{l}\text { Described by Kim } \\
\text { et al. }\end{array}$ & 40.8 & $\begin{array}{c}\text { Clinical, imaging, } \\
\text { pathological, } \\
\text { outcome, histological }\end{array}$ & $\begin{array}{l}\text { OT, EBL, male gender, age, waist } \\
\text { circumference, fat density on CT, } \\
\text { MAP score, and larger adipocytes }\end{array}$ \\
\hline $\begin{array}{l}\text { Shintaro et al. } \\
(2017)\end{array}$ & 92 & $\begin{array}{l}\text { Laparoscopic } \\
\text { donor } \\
\text { nephrectomy }\end{array}$ & $\begin{array}{l}\text { Intraoperative } \\
\text { videos }\end{array}$ & 55.4 & $\begin{array}{l}\text { Clinical, imaging, } \\
\text { outcome, IHC }\end{array}$ & $\begin{array}{c}\text { Perinephric fat area, stranding, sIL-6R, } \\
\text { and OT }\end{array}$ \\
\hline $\begin{array}{l}\text { Khene et al. } \\
\text { (2017) }\end{array}$ & 202 & RPN & Operative records & 39.6 & $\begin{array}{l}\text { Clinical, imaging, } \\
\text { outcome }\end{array}$ & $\begin{array}{c}\text { Male gender, obesity, hypertension, } \\
\text { MAP score, OT, EBL, transfusion, and } \\
\text { conversion to open surgery or radical } \\
\text { nephrectomy }\end{array}$ \\
\hline
\end{tabular}

density can predict the difficulty of renal peritoneal fat separation. The above differences may be related to the measurement method of perinephric fat density. During surgery, we found that perinephric fat only adhered to the surface of the kidney and not to the posterior peritoneum or posterior abdominal wall, suggesting that the adhesion or inflammatory area may be closed to the kidney surface. So, we measure perinephric fat density in a high-density area adjacent to the renal capsule, and we found that some patients with SPF had significantly increased perinephric fat $\mathrm{HU}$ value on enhanced CT. Our results suggest that perinephric fat density may be a complement to the MAP score.

Several authors considered that APF can increase the risk of OT and bleeding during PN $[6,10,14]$. However, our results only find that drain output was associated with APF [27]. The OT and intraoperative EBL may be more affected by surgeon's surgical experience and other reasons, such as location of the tumor, damage of variant renal blood vessels and adjacent organs, and suture cutting off the renal parenchyma. Senior surgeons can speed up the surgical process and reduce damage and intraoperative bleeding, suggesting that whether APF can affect the OT and EBL needs further verification [28].

In this study, we found another new layer of fascia on the lateral side of renal capsule in the NPF group for the first time. This fascia was defined as extracapsular fascia. During $\mathrm{PN}$, perinephric fat can be easily blunt dissected along the gap between renal capsule and extracapsular fascia.
However, due to chronic inflammation for the SPF group, extracapsular fascia was taken place by thickening and fusion of renal capsule with many vessels, which will cause decapsulation and increasing hemorrhage in the process of kidney mobilization.

In conclusion, APF is more prevalent in aging and male populations, particularly in those with hypertension. Our research confirmed that $\mathrm{APF}$ is associated with drain output and accompanied by immune cells gathering in renal cortex near thickened renal capsule which with many vessels. Radiological factors show that perinephric stranding, posterior perinephric fat thickness, and perinephric fat density can be used to predict PFAD.

\section{Data Availability}

No data were used to support this study.

\section{Ethical Approval}

Ethical approval was given by the medical Ethics Committee of Chinese Clinical Trial Registry with the following reference number: ChiCTR-POC-15006412.

\section{Consent}

Not applicable. 


\section{Disclosure}

Junqiang Liu, Yiheng Jiang, Hongwei Huang, Zheng Zhu, and Jing Chen are the co-first authors. This study has been published as preprint (DOI:10.21203/rs.2.21034/v3).

\section{Conflicts of Interest}

The authors declare that they have no conflicts of interest.

\section{Authors' Contributions}

Junqiang Liu, Yiheng Jiang, Hongwei Huang, Zheng Zhu, and Jing Chen contributed equally to this work. In this research, J.L., H.H., and Y.J. designed and performed the experiment. D.L., Z.Z., J.C., and L.W. optimized algorithm flow. H.H and Y.J. wrote, revised, and finalized the article. J.W. and D.Y. reviewed and edited the manuscript. H.Z. and X.S. collected and collated figures. X.W., X.Z., Q.C., and S.N. summarized the statistical data. All authors read and approved the manuscript.

\section{Supplementary Materials}

Representative examples of CD34 and CD45 immunohistological staining. (a) APF CD34 for vascular staining, positive in glomerular while negative in kidney tubules. (b) APF CD45 immune cells in the group, positive in lymph nodes while negative in normal kidney tissues. Scale bar $=80 \mathrm{~mm}$. (Supplementary Materials)

\section{References}

[1] S. C. Campbell, A. C. Novick, A. Belldegrun et al., "Guideline for management of the clinical T1 renal mass," The Journal of Urology, vol. 182, no. 4, pp. 1271-1279, 2009.

[2] S. Maclennan, M. Imamura, M. C. Lapitan et al., "Systematic review of oncological outcomes following surgical management of localised renal cancer," European Urology, vol. 61, no. 5, pp. 972-993, 2012.

[3] S. Maclennan, M. Imamura, M. C. Lapitan et al., "Systematic review of perioperative and quality-of-life outcomes following surgical management of localised renal cancer," European Urology, vol. 62, no. 6, pp. 1097-1117, 2012.

[4] J. R. Bylund, H. Qiong, P. L. Crispen, R. Venkatesh, and S. E. Strup, "Association of clinical and radiographic features with perinephric "sticky" fat," Journal of Endourology, vol. 27, no. 3, pp. 370-373, 2013.

[5] A. J. Davidiuk, A. S. Parker, C. S. Thomas et al., "Mayo adhesive probability score: an accurate image-based scoring system to predict adherent perinephric fat in partial nephrectomy," European Urology, vol. 66, no. 6, pp. 1165-1171, 2014.

[6] Z.-E. Khene, B. Peyronnet, R. Mathieu, T. Fardoun, G. Verhoest, and K. Bensalah, "Analysis of the impact of adherent perirenal fat on peri-operative outcomes of robotic partial nephrectomy," World Journal of Urology, vol. 33, no. 11, pp. 1801-1806, 2015.

[7] A. J. Davidiuk, A. S. Parker, C. S. Thomas, M. G. Heckman, K. Custer, and D. D. Thiel, "Prospective evaluation of the association of adherent perinephric fat with perioperative outcomes of robotic-assisted partial nephrectomy," Urology, vol. 85, no. 4, pp. 836-842, 2015.

[8] L. Martin, O. Rouviere, R. Bezza, J. Bailleux, F. Abbas, and A.-M. Schott-Pethelaz, "Mayo adhesive probability score is an independent computed tomography scan predictor of adherent perinephric fat in open partial nephrectomy," The Journal of Urology, vol. 103, pp. 124-128, 2016.

[9] Y. Zheng, P. Espiritu, T. Hakky, K. Jutras, and P. E. Spiess, "Predicting ease of perinephric fat dissection at time of open partial nephrectomy using preoperative fat density characteristics," BJU International, vol. 114, no. 6, pp. 872-880, 2015.

[10] C. Dariane, T. L. Guilchet, S. Hurel, F. Audenet, A. Beaugerie, and C. Badoual, "Prospective assessment and histological analysis of adherent perinephric fat in partial nephrectomies," Urologic Oncology Seminars \& Original Investigations, vol. 35, no. 2, pp. 39-e9, 2016.

[11] S. Narita, T. Kumazawa, N. Tsuchiya et al., "Host-related risk factors for adherent perinephric fat in healthy individuals undergoing laparoscopic living-donor nephrectomy," Surgical Laparoscopy Endoscopy \& Percutaneous Techniques, vol. 27, no. 4, pp. e69-e73, 2017.

[12] B. H. Eisner, J. Zargooshi, A. D. Berger et al., "Gender differences in subcutaneous and perirenal fat distribution," Surgical and Radiologic Anatomy, vol. 32, no. 9, pp. 879-882, 2010.

[13] S. Kim, S.-K. Choi, S. M. Lee et al., "Predictive value of preoperative unenhanced computed tomography during ureteroscopic lithotripsy: a single institute's experience," Korean Journal of Urology, vol. 54, no. 11, p. 772, 2013.

[14] N. J. Kocher, S. Kunchala, C. Reynolds, E. Lehman, S. Nie, and J. D. Raman, "Adherent perinephric fat at minimally invasive partial nephrectomy is associated with adverse peri-operative outcomes and malignant renal histology," BJU International, vol. 117 , no. 4, pp. 636-641, 2016.

[15] D. D. Thiel, A. J. Davidiuk, C. Meschia et al., "Mayo adhesive probability score is associated with localized renal cell carcinoma progression-free survival," Urology, vol. 89, no. 4, pp. 54-62, 2016.

[16] B. M. Benway, G. Baca, S. B. Bhayani et al., "28 selective versus non-selective arterial clamping for laparoscopic partial nephrectomy: impact upon renal function of a solitary kidney in a porcine model," European Urology Supplements, vol. 8, no. 4, p. 127, 2009.

[17] K. M. Anderson, T. U. Lindler, G. R. Lamberton, P. W. Baron, O. K. Ojogho, and D. D. Baldwin, "Laparoscopic donor nephrectomy: effect of perirenal fat upon donor operative time," Journal of Endourology, vol. 22, no. 10, pp. 2269-2274, 2008.

[18] T. Tchkonia, D. E. Morbeck, T. Von Zglinicki et al., "Fat tissue, aging, and cellular senescence," Aging Cell, vol. 9, no. 5, pp. 667-684, 2010.

[19] Y. Zhu, H.-K. Wang, H.-L. Zhang et al., "Visceral obesity and risk of high grade disease in clinical Tla renal cell carcinoma," The Journal of Urology, vol. 189, no. 2, pp. 447-453, 2013.

[20] S. P. Weisberg, D. Mccann, M. Desai, M. Rosenbaum, R. L. Leibel, and A. W. Ferrante, "Obesity is associated with macrophage accumulation in adipose tissue," Journal of Clinical Investigation, vol. 112, no. 12, pp. 1796-1808, 2003.

[21] G. J. Neels, "Inflamed fat: what starts the fire?" Journal of Clinical Investigation, vol. 116, no. 1, pp. 33-35, 2006.

[22] Y. Kobayashi, H. Kurahashi, Y. Matsumoto et al., "Factors predicting adhesion between renal capsule and perinephric adipose tissue in partial nephrectomy," Acta Medica Okayama, vol. 70, no. 2, pp. 69-74, 2016. 
[23] C. Ji, S. Tang, K. Yang et al., "Analysis of factors influencing Mayo adhesive probability score in partial nephrectomy," Medical Science Monitor, vol. 23, pp. 6026-6032, 2017.

[24] L. C. Evans, G. Petrova, T. Kurth et al., "Increased perfusion pressure drives renal T-cell infiltration in the dahl salt-sensitive rat," Hypertension, vol. 70, no. 3, pp. 543-551, 2017.

[25] O. Timar, F. Sestier, and E. Levy, "Metabolic syndrome X: a review," Canadian Journal of Cardiology, vol. 16, no. 6, pp. 779-89, 2000.

[26] R. Monteiro and I. Azevedo, "Chronic inflammation in obesity and the metabolic syndrome," Mediators of Inflammation, vol. 2010, Article ID 289645, 10 pages, 2010.

[27] A. Brassetti, U. Anceschi, R. Bertolo, M. Ferriero, and G. Simone, "Comprehensive long-term assessment of outcomes following robot-assisted partial nephrectomy for renal cell carcinoma: The ROMe's achievement and its predicting nomogram," 2020.

[28] M. Ferriero, A. M. Bove, G. Tuderti, U. Anceschi, A. Brassetti, and M. Costantini, "Impact of learning curve on perioperative outcomes of off-clamp minimally invasive partial nephrectomy: propensity score matched comparison of outcomes between training versus expert series," Minerva Urologica $e$ Nefrologica = The Italian Journal of Urology and Nephrology, vol. 73, no. 5, 2020. 